\title{
Substrate and strain alter the muscle-derived mesenchymal stem cell secretome to promote myogenesis
}

\author{
Michael De Lisio ${ }^{1,2}$, Tor Jensen ${ }^{3}$, Richard A Sukiennik ${ }^{1,2}$, Heather D Huntsman ${ }^{1,2}$ and Marni D Boppart ${ }^{1,2^{*}}$
}

\begin{abstract}
Introduction: Mesenchymal stem cells (MSCs) reside in a variety of tissues and provide a stromal role in regulating progenitor cell function. Current studies focus on identifying the specific factors in the niche that can alter the MSC secretome, ultimately determining the effectiveness and timing of tissue repair. The purpose of the present study was to evaluate the extent to which substrate and mechanical strain simultaneously regulate MSC quantity, gene expression, and secretome.
\end{abstract}

Methods: MSCs (Sca- $1^{+} \mathrm{CD} 45^{-}$) isolated from murine skeletal muscle (muscle-derived MSCs, or mMSCs) via fluorescence-activated cell sorting were seeded onto laminin (LAM)- or collagen type 1 (COL)-coated membranes and exposed to a single bout of mechanical strain (10\%, $1 \mathrm{~Hz}, 5$ hours).

Results: mMSC proliferation was not directly affected by substrate or strain; however, gene expression of growth and inflammatory factors and extracellular matrix (ECM) proteins was downregulated in MMSCs grown on COL in a manner independent of strain. Focal adhesion kinase (FAK) may be involved in substrate regulation of mMSC secretome as FAK phosphorylation was significantly elevated 24 hours post-strain in mMSCs plated on LAM but not $\mathrm{COL}(P<0.05)$. Conditioned media $(C M)$ from mMSCs exposed to both LAM and strain increased myoblast quantity 5.6-fold 24 hours post-treatment compared with myoblasts treated with serum-free media $(P<0.05)$. This response was delayed in myoblasts treated with CM from mMSCs grown on COL.

Conclusions: Here, we demonstrate that exposure to COL, the primary ECM component associated with tissue fibrosis, downregulates genes associated with growth and inflammation in mMSCs and delays the ability for mMSCs to stimulate myoblast proliferation.

\section{Introduction}

Mesenchymal stem/stromal cells (MSCs) are a pluripotent population of cells that reside in a variety of tissues throughout the body. These cells are defined by their capacity for multi-lineage differentiation, including chondrogenesis, osteogenesis, and adipogenesis [1]. Owing to their multi-lineage potential, immune-privileged nature, relative ease of isolation, and ability to be expanded in culture, MSCs have received much attention for their potential use in cell therapy [2]. Recently, it was suggested that the

\footnotetext{
* Correspondence: mboppart@illinois.edu

${ }^{1}$ Department of Kinesiology and Community Health, University of Illinois, Urbana, IL, USA

${ }^{2}$ Beckman Institute for Advanced Science and Technology, University of Illinois, 405 N. Mathews Avenue, MC-251, Urbana, IL 61801, USA

Full list of author information is available at the end of the article
}

primary mechanism by which MSCs contribute to tissue repair is indirect via secretion of factors that stimulate native tissue repair processes or tissue-resident stem cells [3]. It was suggested that the MSC secretome is strongly regulated by the local microenvironment [3]. For example, hypoxia can stimulate MSC secretion of vascular endothelial growth factor (VEGF) and interleukin-6 (IL-6) [4], and factors released from MSCs can reverse degenerative processes in a variety of tissues, including heart [3], brain [5], the hematopoietic system [6], and skeletal muscle [7]. For these reasons, MSCs provide an exciting cell population for therapy, and defining cell culture conditions that allow optimal MSC expansion and function prior to

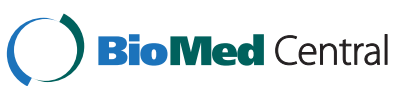

(c) 2014 De Lisio et al.; licensee BioMed Central Ltd. This is an Open Access article distributed under the terms of the Creative Commons Attribution License (http://creativecommons.org/licenses/by/4.0), which permits unrestricted use, distribution, and reproduction in any medium, provided the original work is properly credited. The Creative Commons Public Domain Dedication waiver (http://creativecommons.org/publicdomain/zero/1.0/) applies to the data made available in this article, unless otherwise stated. 
transplantation may be an effective strategy to enhance their efficacy.

Mesenchymal progenitor cells have been identified in skeletal muscle that directly or indirectly contributes to repair in response to injury [7]. These multi-potent stem cells have been isolated by using unique cell surface markers and thus classified as side population cells $[8,9]$, pericytes [10], muscle-derived stem cells [11], musclederived MSCs (mMSCs) [12,13], fibro/adipogenic progenitors [14], and $\mathrm{PW}^{+}$interstitial cells [15], and some degree of overlap likely exists between these cell populations. Whereas some of these cells can become myogenic, the majority have limited capacity for myogenic differentiation and are primed to secrete factors essential for indirect repair of skeletal muscle. We recently defined a population of MSCs, identified by expression of stem cell antigen 1 $\left(\mathrm{Sca}-1^{+}\right)$and lack of expression of a hematopoietic cell surface marker $\left(\mathrm{CD} 45^{-}\right)$, that is capable of osteogenic, chondrogenic, and adipogenic differentiation and accumulate in skeletal muscle ( $m \mathrm{MSCs})$ after eccentric exercise in an $\alpha 7-$ integrin-dependent manner [12]. We also recently demonstrated that Sca- $1^{+} \mathrm{CD} 45^{-} \mathrm{mMSCs}$ are involved in muscle repair after injury [12], perhaps through secretion of paracrine factors [16]. Currently, minimal information exists regarding the cues in the environment that regulate MSC paracrine factor synthesis and secretion [16].

The MSC niche is complex, and composition of the MSC niche in each tissue type is defined by a unique combination of growth/inflammatory factors, extracellular matrix (ECM) components, other cell types, and topography [17]. In skeletal muscle, the extracellular environment consists of a variety of matrix proteins, including but not limited to collagen type 1 (COL) and laminin (LAM) [18]. COL is the primary collagen isoform in the perimysium and accumulates during muscle fibrosis [18] that accompanies disease, injury, and aging $[19,20]$. The resulting fibrosis causes dysregulation of muscle-derived stem cells which may be a major factor inhibiting muscle regeneration and repair [19,21]. Because the musculoskeletal system is highly dynamic and responsive to movement, mechanical strain may largely intersect and alter the MSC response to COL or LAM or both [22]. Some information exists regarding MSC mechanosensory signaling and lineage specification. For example, MSCs derived from bone marrow are directed toward an osteogenic fate when subjected to multiple bouts of biaxial strain [23-25] and this may be dependent on the activation of focal adhesion kinase (FAK) [10]. In addition, mMSCs secrete a variety of growth factors in response to biaxial strain in the presence of LAM [16]. However, the full extent to which MSCs residing in skeletal muscle are responsive to mechanical strain in the context of substrate has yet to be determined. This information is essential for elucidating a role for mMSCs in whole tissue health and designing cell- based therapies that can most effectively combat skeletal muscle disease and disabilities.

The purpose of the present investigation was to characterize the mMSC response to mechanical strain in the context of substrate. We hypothesized that mechanical strain would increase mMSC quantity or gene expression of factors that would promote skeletal muscle regeneration (or both) while exposure to COL would inhibit this response.

\section{Materials and methods}

\section{a7 Integrin transgenic mice}

Mice created to overexpress $\alpha 7$ integrin specifically in skeletal muscle (MCK- $\alpha 7 \mathrm{BX} 2$ ), referred to as $\alpha 7 \mathrm{Tg}$ mice, were produced and bred in-house [26]. Overexpression of $\alpha 7$ integrin BX2 subunit was determined by polymerase chain reaction (PCR) analysis (primers: MCK1, 5' $-\mathrm{CA}$ AGCTGCACGCCTGGGTCC-3' and AATII, 5' -GGCAC CCATGACGTCCAGATTGAAG-3') as previously described [27]. Mice were maintained on a 12-hour light/ dark cycle and were provided food and water ad libitum. All protocols were approved by the Institutional Animal Care and Use Committee of the University of Illinois at Urbana-Champaign.

\section{Muscle-derived mesenchymal stem/stromal cell isolation} mMSCs were isolated as previously described [12,16]. Briefly, 5- to 6-week-old $\alpha 7 \mathrm{Tg}$ mice were sacrificed by carbon dioxide $\left(\mathrm{CO}_{2}\right)$ asphyxiation, and gastrocnemiussoleus complexes were resected and minced into a fine paste. Minced muscle tissue was enzymatically digested in phosphate-buffered saline containing $0.2 \%$ collagenase Type 2 (Worthington, Biochemical Corp., Lakewood, NJ, USA), $60 \mathrm{U} / \mathrm{mL}$ DNase (Sigma-Aldrich, St. Louis, MO, USA), and $2.5 \mathrm{mM} \mathrm{Ca}_{2} \mathrm{Cl}_{2}$ at $37^{\circ} \mathrm{C}$ for 45 minutes. Muscle slurries were mixed with Hank's Balanced Salt Solution containing $20 \%$ fetal bovine serum (FBS) and $0.1 \%$ penicillin/streptomycin to stop enzymatic digestion and were passed through a 70- $\mu \mathrm{m}$ filter. Cells were blocked in Fcreceptor block (CD16/CD32; eBioscience, San Diego, CA, USA) for 10 minutes on ice and then stained with Sca-1$\mathrm{PE}$ and CD45-APC (eBioscience). After washing, cells were passed through a $40-\mu \mathrm{m}$ filter prior to sorting on an iCyt Reflection System (Champaign, IL, USA). Unstained and single-stained controls were used to establish gates. Sorted cells were seeded onto plastic cell culture dishes at a density of $2.5 \times 10^{4}$ cells per $\mathrm{cm}^{2}$ in growth media (Dulbecco's modified Eagle's medium [DMEM]/10\% FBS/ $5 \mu \mathrm{g} / \mathrm{mL}$ gentamicin). After 3 days of culture at $37^{\circ} \mathrm{C}$ and $5 \% \mathrm{CO}_{2}$, non-adherent cells were removed.

In vitro strain protocol and assessment of muscle-derived mesenchymal stem/stromal cell quantity

Passage- 1 or -2 mMSCs were seeded at equal density on either laminin (YIGSR; LAM)- or collagen type 1 (COL)- 
coated Flexcell plates (Flexcell International Corporation, McKeesport, PA, USA). Cells were allowed to adhere and expand for 12 to 72 hours in growth media until they were approximately $80 \%$ confluent. Cells were strained according to our previously published protocol that has been shown to alter myogenic gene expression in mMSCs [12] and the mMSC secretome in a manner that supported arteriogenesis [16]. Briefly, cells were exposed to $10 \%$ biaxial strain at a frequency of $1 \mathrm{~Hz}$ for 5 hours using an FX-4000 Flexercell Tension System (Flexcell International Corporation). Non-strained cells were maintained in the same manner but not exposed to strain. Four experimental conditions were tested: (1) LAM/No Strain (NSL), (2) LAM/Strain (SL), (3) COL/No Strain (NSC), and (4) COL/Strain (SC). For conditioned media $(\mathrm{CM})$ experiments, growth media were replaced with serum-free media (SFM) (DMEM/ $5 \mu \mathrm{g} / \mathrm{mL}$ gentamicin) prior to the strain protocol, collected at 24 hours after the initiation of strain, and stored at $-80^{\circ} \mathrm{C}$ until use. mMSCs were trypsinized and quantified by using a hemocytometer 48 hours after strain. All images were obtained by using a Zeiss AxioCam digital camera and Axiovision software at 20x magnification (Zeiss, Thornwood, NY, USA).

\section{Gene expression analysis}

Three hours after stretch, mMSCs were collected by cell scraping and were centrifuged, and the pellet was frozen at $-80^{\circ} \mathrm{C}$. Isolation of total RNA was conducted by using the PureLink RNA mini kit (Ambion, a brand of Life Technologies, Carlsbad, CA, USA) in accordance with the instructions of the manufacturer. The RNA was quantified spectrophotometrically via the Synergy H1 Hybrid MultiMode Microplate Reader (Biotek, Winooski, VT, USA). Reverse transcription of RNA to cDNA was completed by using a High Capacity cDNA RT Kit (Applied Biosystems, Grand Island, NY). Quantitative real-time polymerase chain reaction (qPCR) was performed either by using SYBR Green/Rox master mix (SuperArray Biosciences, Frederick, $\mathrm{MD}$, USA) with previously published primer sequences or by using pre-designed Taqman primers and probes validated by Applied Biosystems (Foster City, CA, USA) (Tables 1 and 2). qPCR was performed by using the ABI $7900 \mathrm{HT}$ Fast Real Time PCR System (Applied Biosystems) under standard conditions and analyzed by using SDS 2.4 Software (Applied Biosystems). Dissociation curve analysis was performed for SYBR Green reactions to confirm primer specificity. Changes in gene expression relative to LAM/NS were calculated by using the $2^{-\Delta \Delta C T}$ method [28] with GAPDH or L32 as housekeeping genes.

\section{Western blotting}

Proteins were extracted directly from cell culture plates in ice-cold lysis buffer consisting of $50 \mathrm{mM}$ Tris- $\mathrm{HCl}$,
Table 1 Primer pairs for SYBR Green analysis

\begin{tabular}{lll}
\hline Gene & Forward & Reverse \\
\hline Myf5 & TGAAGGATGGACATGACGGACG & TTGTGTGCTCCGAAGGCTGCTA \\
MyoD & TACCCAAGGTGAGATCCTG & CATCATGCCATCAGAGCAGT \\
Myogenin & GAGACATCCCCCTATTTCTACCA & GCTCAGTCCGCTCATAGCC \\
Pax7 & CTGGATGAGGGCTCAGATGT & GGTTAGCTCCTGCCTGCTTA \\
L32 & TCCACAATGTCAAGGAGCTG & ACTCATTITTCGCTGCGT \\
COL1a2 & GCAGGTCACCTACTCTGTCCT & CTTGCCCCATTCATTTGTT \\
Laminin a1 & CAGCGCCAATGCTACCTGT & GGATTCGTACTGTACCGTCACA \\
Laminin a2 & TCCCAAGCGCATCAACAGAG & CAGTACATCTCGGGTCCTITTC \\
GM-CSF & ACCACCTATGCGGATTTCAT & TCATTACGCAGGCACAAAAG \\
HGF & ATGTGGGGGACCAAACTTCTG & GGATGGCGACATGAAGCAG \\
\hline
\end{tabular}

$150 \mathrm{nM} \mathrm{NaCl}, 1 \mathrm{mM}$ PMSF, 1\% NP-40, 0.5\% sodium deoxycholate, and $0.1 \%$ SDS with protease and phosphatase inhibitors (Roche, Indianapolis, IN, USA). Samples were incubated in lysis buffer for 45 minutes at $4^{\circ} \mathrm{C}$, and supernatants were collected for determination of protein concentrations by Bradford assay. Equal amounts of protein, as confirmed by ponceau S, in $4 \mathrm{X}$ Laemlli buffer were separated by SDS-PAGE and transferred to nitrocellulose membranes. After blocking with $5 \%$ non-fat dry milk or $5 \%$ bovine serum albumin, membranes were incubated in the following primary antibodies: phospho-mTOR (Ser2448), mTOR, p70 S6 Kinase, phospho-p70 S6 Kinase (Thr389), phospho-FAK (Tyr 397), phospho-NF-кB p65 (Ser 536) (Cell Signaling Technology, Boston, MA, USA), FAK (Santa Cruz Biotechnology, Santa Cruz, CA, USA), or NF-kB p65 (eBioscience) overnight at $4^{\circ} \mathrm{C}$. Horseradish peroxidase-conjugated anti-rabbit secondary antibody (Jackson ImmunoResearch Laboratories, Inc., West Grove, PA, USA) was applied for 1 hour at room temperature. Proteins were detected with SuperSignal West Pico Chemiluminescent Substrate (Pierce, Rockford, IL, USA) by using a Bio-Rad ChemiDoc XRS system (Bio-Rad Laboratories, Hercules, CA, USA). After detection of phosphorylated proteins, membranes were stripped with Restore Western Blot Stripping Buffer (Thermo Fisher Scientific, Waltham, MA, USA) prior to probing for total protein. Protein content was quantified by using Quantity One software

Table 2 Primers and probes for Taqman analysis

\begin{tabular}{lll}
\hline Gene & Assay ID & Gene ID \\
\hline VEGFa & Mm01281449_m1 & 22339 \\
IL-6 & Mm00446190_m1 & 16193 \\
TGF- $\beta 1$ & Mm01178820_m1 & 21803 \\
TNF-a & Mm00443258_m1 & 21926 \\
COL1a1 & Mm00801666_g1 & 12842 \\
GAPDH & Mm03302249_g1 & 14433 \\
\hline
\end{tabular}


(Bio-Rad Laboratories) with background subtracted. Any samples that did not give values above background were omitted from analysis. For each protein, phosphorylated protein content was expressed relative to total protein content and normalized to the average of LAM/NS to obtain a fold change.

\section{Myoblast and myotube culture experiments}

Littermates of $\alpha 7 \mathrm{Tg}$ mice that did not overexpress the transgene were used for primary myoblast isolation. Myoblasts were isolated from neonatal mice by using a sequential plating technique as previously described [29], except plates were coated with gelatin instead of collagen as we have found that gelatin increases the efficiency of isolation. Primary myoblasts and $\mathrm{C} 2 \mathrm{C} 12$ cells (ATCC, Manassas, VA, USA) were maintained at $37^{\circ} \mathrm{C}$ in $5 \% \mathrm{CO}_{2}$, expanded in growth media (DMEM/20\% FBS/ $1 \%$ penicillin-streptomycin), and differentiated in differentiation media (DMEM/2\% horse serum/1\% penicillinstreptomycin). For CM experiments, CM was quickly thawed, centrifuged at $400 \mathrm{~g}$ for 5 minutes at $4^{\circ} \mathrm{C}$ to remove any remaining cells, and added to primary myoblasts seeded at equal density or myotubes formed from C2C12 myoblasts. Myoblasts or myotubes treated with SFM and either growth or differentiation media served as negative and positive controls. Myotubes were harvested 3 hours after treatment with $C M$ for evaluation of hypertrophic signaling. Myoblast quantity was analyzed at 24 and 48 hours post-treatment with $C M$ via the MTT (3-[4,5-dimethylthiazol-2-yl]-2,5-diphenyltetrazolium bromide; thiazolyl blue)-based Cell Growth Determination Kit (Sigma-Aldrich) in accordance with the instructions of the manufacturer, and values were expressed as a fold change relative to myoblasts treated with SFM for the same amount of time.

\section{Statistical analysis}

Data are presented as mean \pm standard error of the mean. Two-factor analysis of variance (substrate $\times$ strain) was conducted followed by Tukey post hoc test if a significant difference was detected. All statistical analyses were conducted by using SigmaPlot 12.5 (Systat Software, Inc., Chicago, IL, USA). Differences were considered significant at a $P$ value of less than 0.05 .

\section{Results}

Exposure to different substrates or strain does not alter muscle-derived mesenchymal stem/stromal cell quantity but alters myogenic gene expression

mMSCs displayed their characteristic fibroblast-like appearance with numerous projections spreading out from the cell body on both COL and LAM (Figure 1A). Neither substrate nor strain influenced the quantity of mMSCs at 48 hours (Figure 1B). Gene expressions of the myogenic regulatory factors (MRFs) Myf5 $(P<0.01)$, MyoD $(P<0.01)$, and Myogenin $(P<0.05)$ were all significantly downregulated in mMSCs grown on COL (Figure 1C-E). The myoblast-specific transcription factor Pax7 was not reliably detected in any samples analyzed (data not shown).

\section{Muscle-derived mesenchymal stem/stromal cell gene expression is downregulated on collagen 1}

Growth factor gene expression was generally downregulated in mMSCs grown on COL with a significant decrease in the expression of VEGFa (Figure 2A; $P<0.01$ ), a trend toward a decrease in expression of granulocyte-macrophage colony-stimulating factor (GM-CSF) (Figure 2B; $P=0.087$ ), and no change in hepatocyte growth factor (HGF) expression (Figure $2 \mathrm{C}$ ). Similarly, gene expression of the inflammatory cytokines IL-6 $(P<0.01)$, transforming growth factor-beta 1 (TGF- $\beta 1) \quad(P<0.05)$, and tumor necrosis factor-alpha $($ TNF- $\alpha)(P<0.01)$ was significantly decreased in mMSCs grown on COL (Figure 2D-F). The expression of growth or inflammatory factors was not influenced by strain (Figure 2A-F). Laminin $\alpha 1$ gene expression was significantly decreased (Figure $3 \mathrm{~A} ; P<0.01$ ) in mMSCs on COL, whereas laminin $\alpha 2$ was not altered (Figure 3B). There was a strong trend for an interaction $(P=0.050)$ between substrate and strain for collagen $1 \alpha 1$ (Figure 3C) with no differences in expression of collagen $1 \alpha 2$.

\section{Substrate and stretch alter the activation of focal adhesion kinase but not nuclear factor-kappa B}

FAK is an intracellular signaling molecule that responds to various factors in the cellular microenvironment and may facilitate the process of mechanotransduction [30]. Levels of phosphorylated FAK were not altered on either substrate 3 hours post-strain (Figure 4A). At 24 hours post-strain, FAK phosphorylation was significantly increased in mMSCs strained on LAM (Figure 4B; $P<0.05$ ), whereas total levels of FAK were increased on COL (data not shown). Neither NF-kB phosphorylation nor levels of total protein were altered by substrate or strain at either time point (Figure 4C-D).

The muscle-derived mesenchymal stem/stromal cell secretome increases myoblast quantity but does not influence hypertrophic signaling in myotubes

CM were collected from mMSCs not strained on LAM (NSL), strained on LAM (SL), not strained on $\mathrm{COL}$ (NSC), and strained on COL (SC) and used to treat $\mathrm{C} 2 \mathrm{C} 12$-derived myotubes or primary myoblasts. CM from SL increased the quantity of primary myoblasts after 24 hours of treatment as compared with NSL and SC (Figure 5A; $P<0.01$ and $P<0.001$, respectively). After 48 hours of treatment, primary myoblasts treated with $\mathrm{CM}$ from mMSCs grown on COL were 

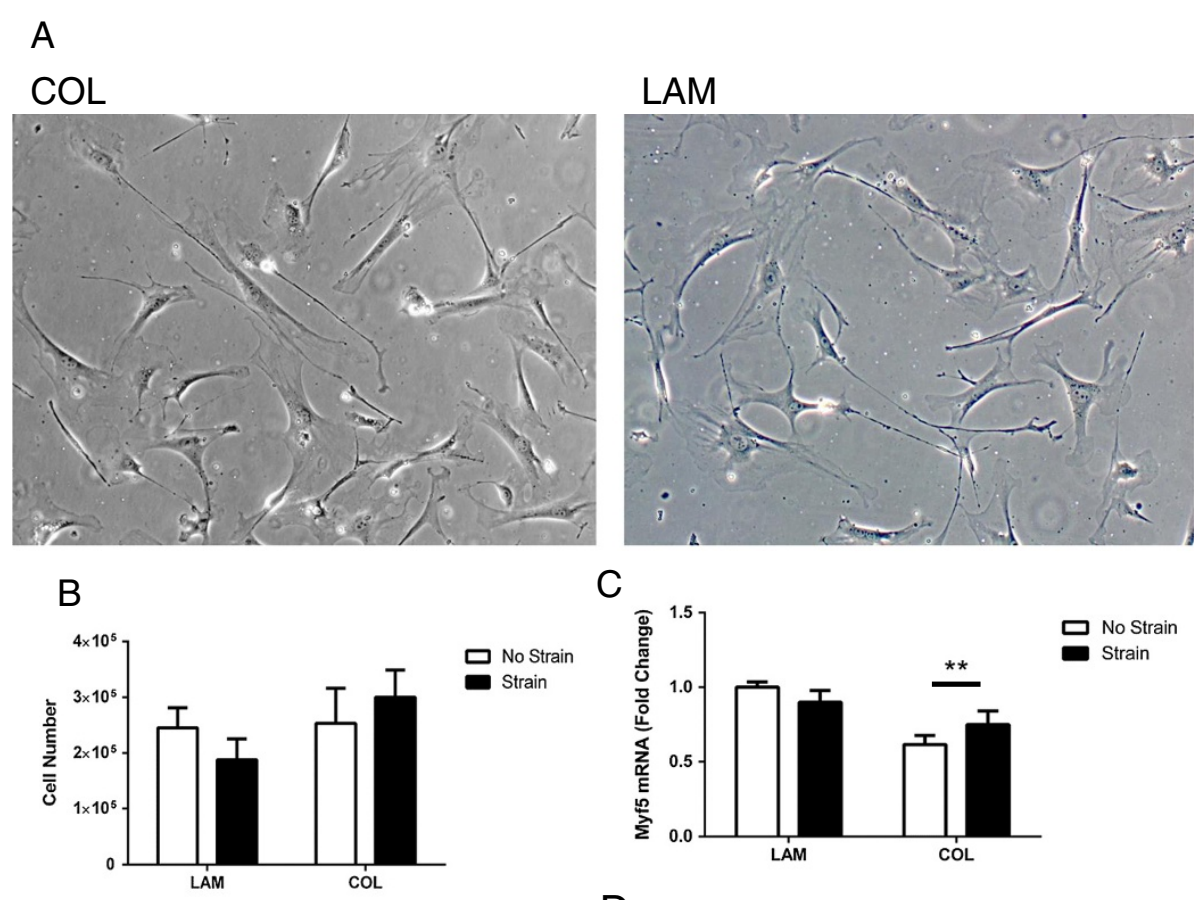

C
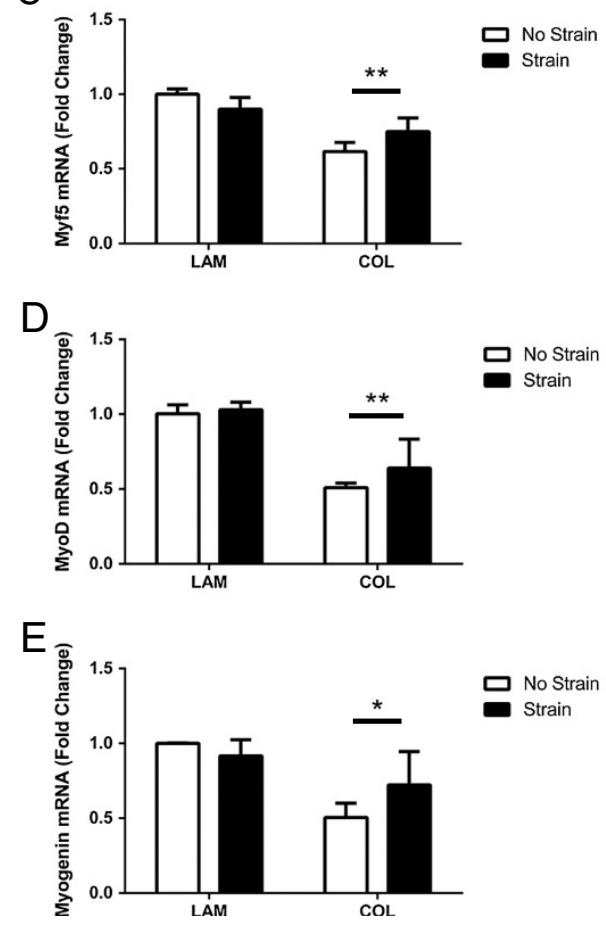

Figure 1 Muscle-derived mesenchymal stem/stromal cell (mMSC) appearance, proliferation, and myogenic gene expression. mMSCs were plated on laminin (LAM) or collagen $(\mathrm{CO})$ and were either exposed to mechanical strain or remained unstrained. Representative images of mMSCs exposed to the various substrates (A). mMSC quantity as determined by hemocytometer 48 hours post-strain with non-strained cells serving as controls ( $n=6$ per group) (B). Gene expression of the myogenic regulatory factors Myf5 (C), MyoD (D), and Myogenin (E) 3 hours post-strain ( $n=3$ per group). Pax7 mRNA was not reliably detected in any group. Fold change is relative to LAM no strain. Data are presented as mean \pm standard error of the mean. ${ }^{*} P<0.01$ versus LAM (main effect for substrate).

significantly increased in quantity (Figure $5 \mathrm{~B} ; P<0.001$ main effect for COL) and a strong trend was observed for increased quantity of primary myoblasts when exposed to CM from strained mMSCs $(P=0.050$ for strain). In myotubes, activation of two signaling molecules involved in the initiation of protein synthesis, mTOR and p70 S6 Kinase, was not altered at 3 hours when exposed to $\mathrm{CM}$ from any of the above conditions (Figure 6A and B).

\section{Discussion}

In the present investigation, we demonstrate that mMSCs are responsive to different substrates in their environment and, to a lesser extent, mechanical strain. $\mathrm{COL}$, an ECM protein that accumulates in fibrotic conditions, resulted in decreased expression of a wide variety of genes, including MRFs, growth factors, cytokines, and ECM proteins in mMSCs. These results suggest that mMSC interaction with collagen decreases their 

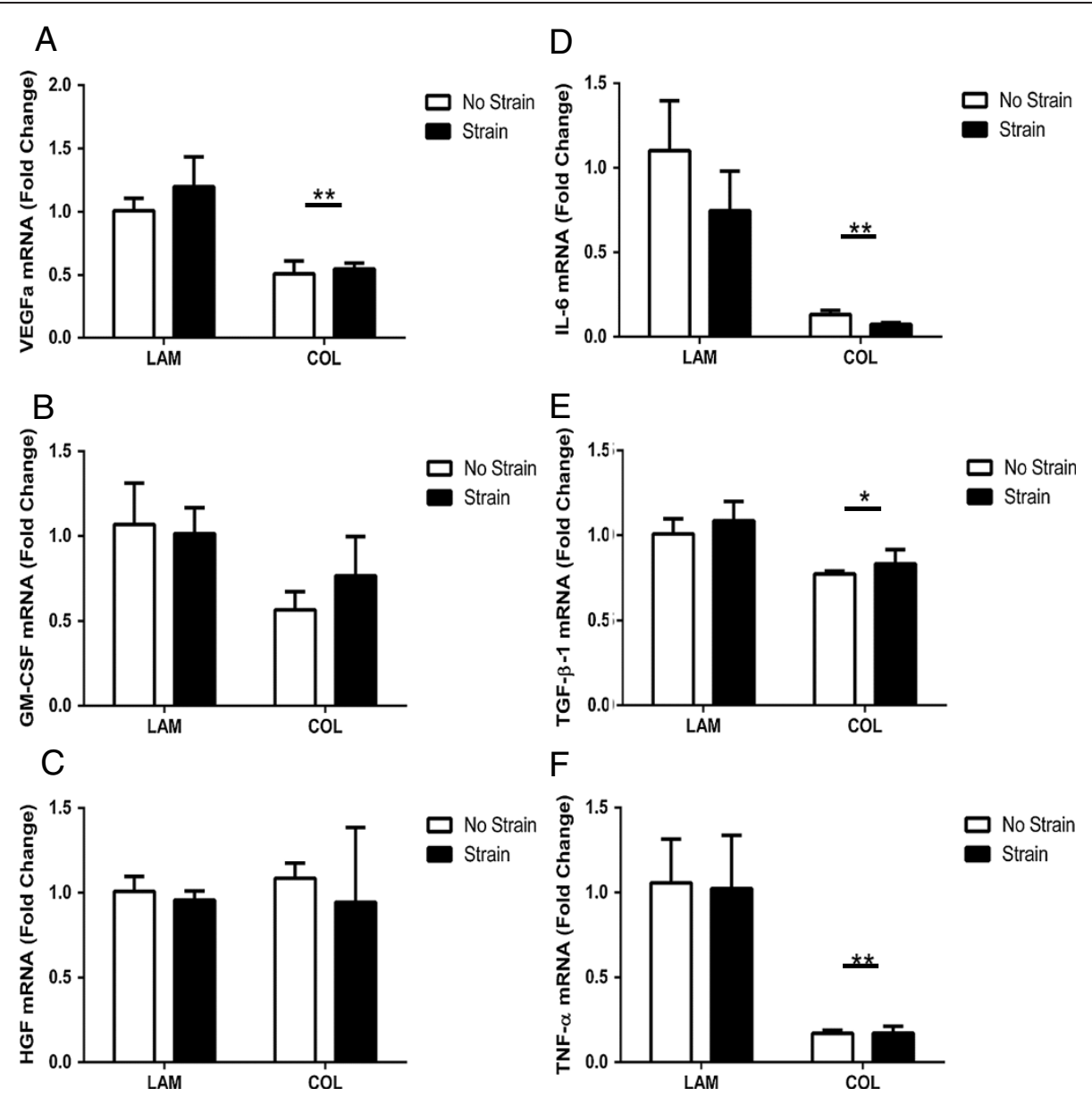

F

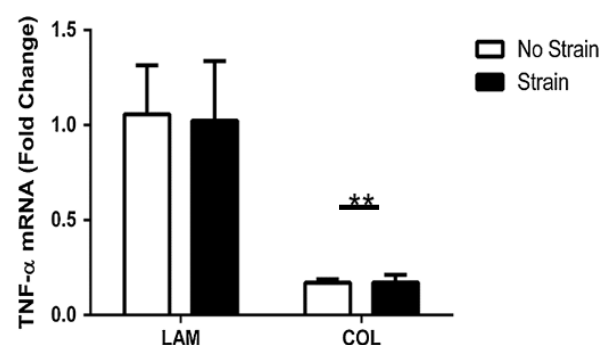

Figure 2 Muscle-derived mesenchymal stem/stromal cell (mMSC) growth and inflammatory factor gene expression. mMSCs were plated on laminin (LAM) or collagen (COL) and were either exposed to mechanical strain or remained unstrained. mRNA for vascular endothelial growth factor a (VEGFa) (A), granulocyte-macrophage colony-stimulating factor (GM-CSF) (B), hepatocyte growth factor (HGF) (C), interleukin-6 (IL-6) (D), transforming growth factor-beta 1 (TGF-B1) (E), and tumor necrosis factor-alpha (TNF-a) (F) was analyzed 3 hours after strain and expressed relative to LAM no strain ( $n=3$ per group). Data are presented as mean \pm standard error of the mean. ${ }^{*} P<0.05 ;{ }^{* *} P<0.01$ versus $L A M$.

potential for myogenic differentiation and decreases their production of growth and inflammatory factors involved acutely in muscle repair or chronically in muscle degeneration. Our CM experiments demonstrated that media from mMSCs strained on LAM enhanced myoblast proliferation at 24 hours while this response was delayed to 48 hours in myoblasts treated with CM from mMSCs grown on COL. Overall, these data suggest that collagen accumulation in fibrotic conditions may impair the myogenic response by inhibiting mMSC function.

mMSCs exist in limited quantities in vivo; therefore, identifying factors that promote expansion or enhance their function would increase the clinical utility of these cells. In agreement with previous findings in primary myoblasts [31], we did not observe any differences in mMSC quantity when grown on LAM or COL. Furthermore, strain did not enhance mMSC proliferation on either substrate. mMSCs may contribute to muscle regeneration or repair by direct differentiation or fusion with existing muscle fibers or indirectly via the promotion of satellite cell activation and differentiation [32]. Expression of the MRFs Myf5, MyoD, and myogenin was downregulated on COL, suggesting that direct differentiation toward the myogenic lineage was suppressed. This is in agreement with a previous study that examined the effects of different substrates on expression of Myf5 as well as myotube formation of a mixed population of primary myoblasts and demonstrated an increase in both of these parameters in cells plated on laminin [31]. Furthermore, it has been suggested that osteogenic differentiation of MSCs is promoted on collagen $1[33,34]$, supporting the notion that COL inhibits myogenic differentiation. Pax7 expression was also not reliably detected in any group and this is in agreement with a previous study demonstrating low Pax7 expression in MSCs [35] and with a previous study from our 
A
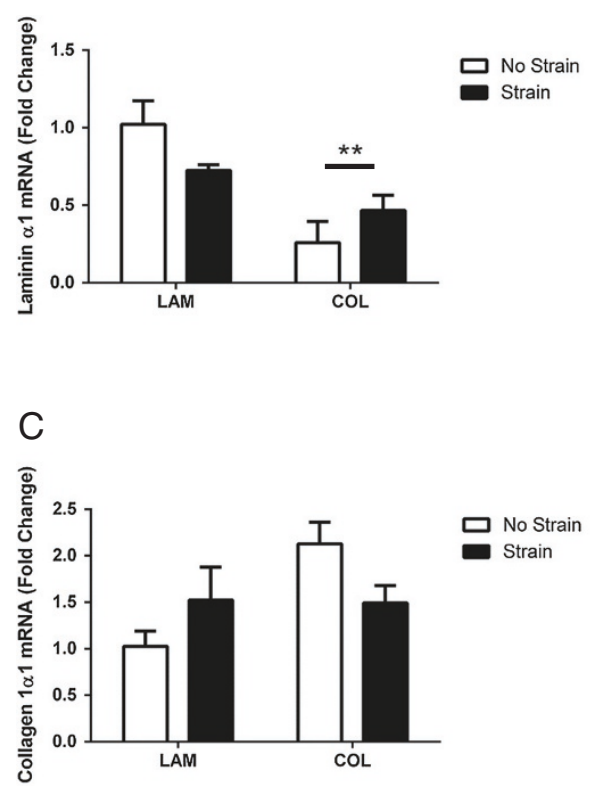

B

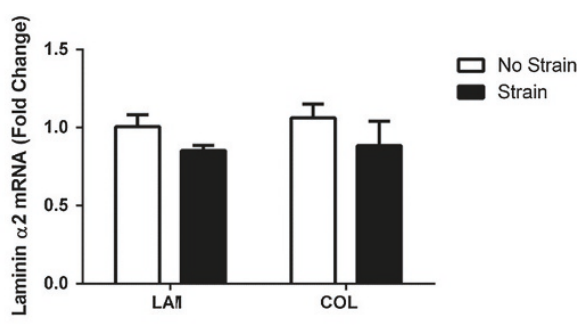

D

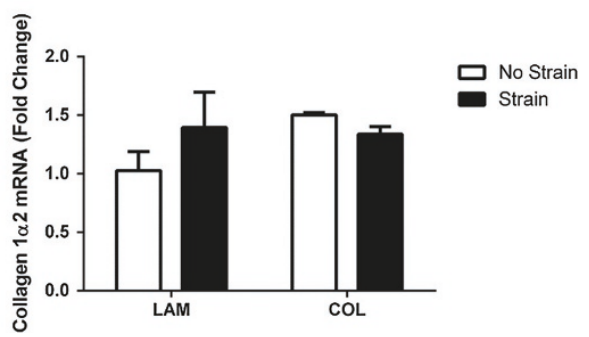

Figure 3 Muscle-derived mesenchymal stem/stromal cell (mMSC) extracellular matrix gene expression. mMSCs were plated on laminin (LAM) or collagen (COL) and were either exposed to mechanical strain or remained unstrained. mRNA for laminin a1 (A), laminin a2 (B), collagen 1 a1 (C), and collagen $1 a 2$ (D) was analyzed 3 hours after strain and expressed relative to LAM no strain ( $n=3$ per group). Data are presented as mean \pm standard error of the mean. ${ }^{* *} P<0.01$ versus LAM.

group [12]. Together, these data suggest that LAM maintains the expression of genes required for myogenesis, while COL downregulates these genes and promotes differentiation along other lineages. The pro-myogenic response on LAM may be related to VEGF signaling as inhibition of VEGF induction in response to mechanical strain decreased the myogenic potential of muscle-derived stem cells (MDSCs) [36], and VEGF expression was significantly downregulated in mMSCs on COL.

Similar to expression of the MRFs and VEGF, gene expression of several inflammatory factors was downregulated on COL. The effects of inflammatory factors on myogenesis are complex with chronic and acute exposures having differential effects. The role of IL-6 on myogenesis has been extensively evaluated with ablation of IL-6 linked to impaired muscle hypertrophy [37], and myoblasts treated with IL-6 in vitro displayed increased differentiation [38]. In addition, IL-6 localizes to human satellite cells from young individuals acutely following eccentric damage $[39,40]$, while this response is dysfunctional with age and may contribute to decreased muscle repair [41]. Acutely, increases in IL-6 and TNF- $\alpha$ can promote myogenesis as TNF- $\alpha$ increased satellite cell proliferation in vivo and in vitro [42]. The pattern of TNF- $\alpha$ expression mirrors that of IL-6, suggesting that these two factors are similarly regulated. On the other hand, chronic exposure to inflammatory cytokines, including IL- 6 and TNF- $\alpha$, has been shown to have detrimental effects on myogenesis [43]. Elevated systemic levels of IL-6 have been associated with muscle wasting in cancer [44] and aging [45]. The present study indicates that mMSCs are a rich source of paracrine factors and that expression of these factors is downregulated on COL. Downregulation of inflammatory factor production on COL may be a protective mechanism to inhibit chronic upregulation of these factors in fibrotic environments that would further contribute to muscle wasting. Further support for this notion is seen in the trend for a decrease $(P=0.087)$ in GM-CSF expression in mMSCs on COL. GM-CSF is involved in the initial repair response of skeletal muscle to injury or mechanical strain through the recruitment of inflammatory cells [46]. If mMSCs recognize COL as a signal for fibrosis or a repaired wound, then decreased recruitment of inflammatory mediators would be a beneficial response for inhibiting propagation of the inflammatory signal.

Aside from altering the paracrine factor composition within the muscle microenvironment, mMSCs may restructure the ECM. Differential expression of laminin $\alpha 1$ and collagen $1 \alpha 1$ was observed in mMSCs plated on different substrates. Laminin $\alpha 1$, a developmentally regulated, critical component of the laminin trimer [47], was downregulated in mMSCs exposed to COL. Laminin$\alpha 1 \beta 1 \gamma 1$ (LM-111) has previously been used as an effective 

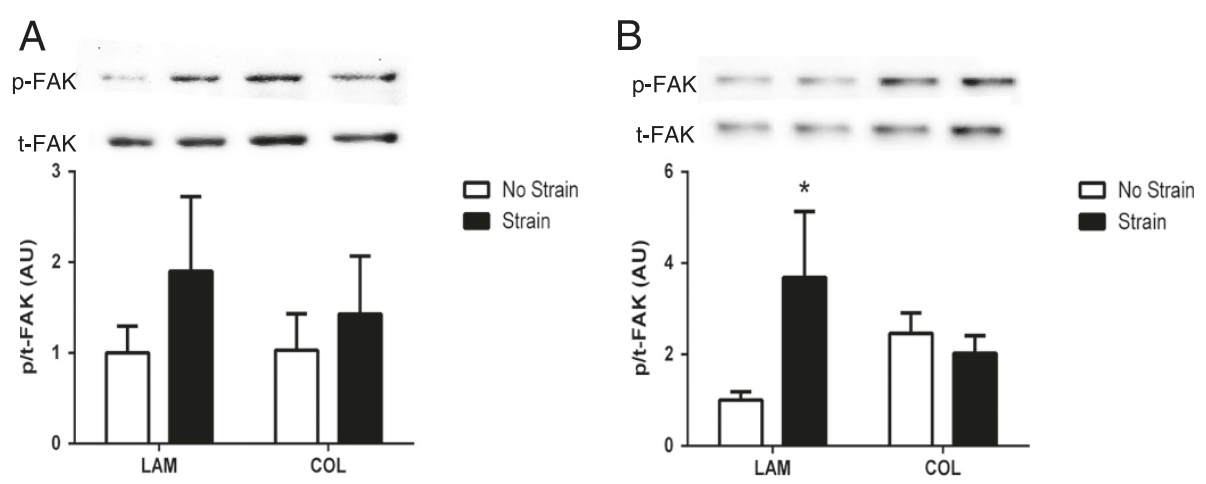

C
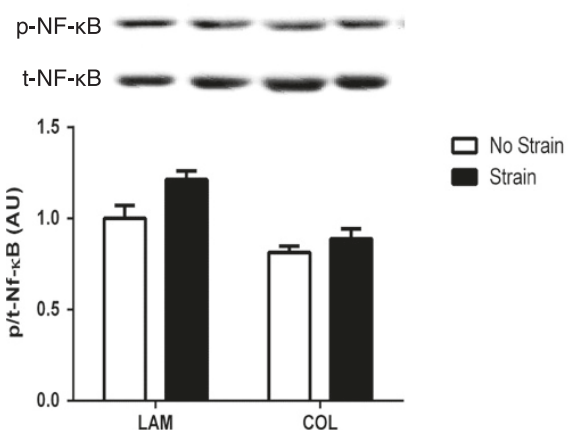

D
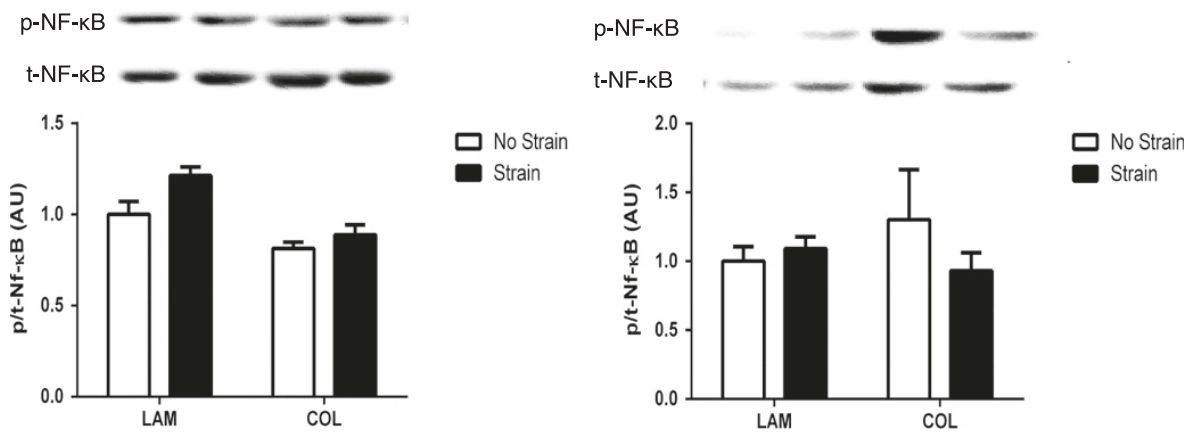

Figure 4 Signaling in muscle-derived mesenchymal stem/stromal cell (mMSCs). mMSCs were plated on laminin (LAM) or collagen (COL) and were either exposed to mechanical strain or remained unstrained. mMSCS were harvested and activation of focal adhesion kinase (FAK) was determined at 3 hours (A) and 24 hours (B) post-strain. Activation of nuclear factor-kappa B (NF-kB) was also analyzed at 3 hours (C) and 24 hours (D) post-strain. Representative images are shown above each respective blot. Data are presented as mean \pm standard error of the mean. ${ }^{*} P<0.05$ versus No Strain/LAM. AU, arbitrary units.

therapy in pre-clinical models for the treatment of muscular dystrophies [48-50], indicating that LAM may improve the muscle microenvironment and increase muscle repair in pathological conditions where attachment of muscle fibers to the basal lamina is impaired. The precise mechanism by which LM-111 can promote regeneration in healthy adult muscle is not currently known. Interestingly, the only mRNA in this study that displayed some regulation by mechanical strain was collagen $1 \alpha 1$, which exhibited a strong trend for an interaction $(P=0.050)$, increasing on LAM with strain and decreasing on COL with strain. The upregulation of collagen $1 \alpha 1$ in response
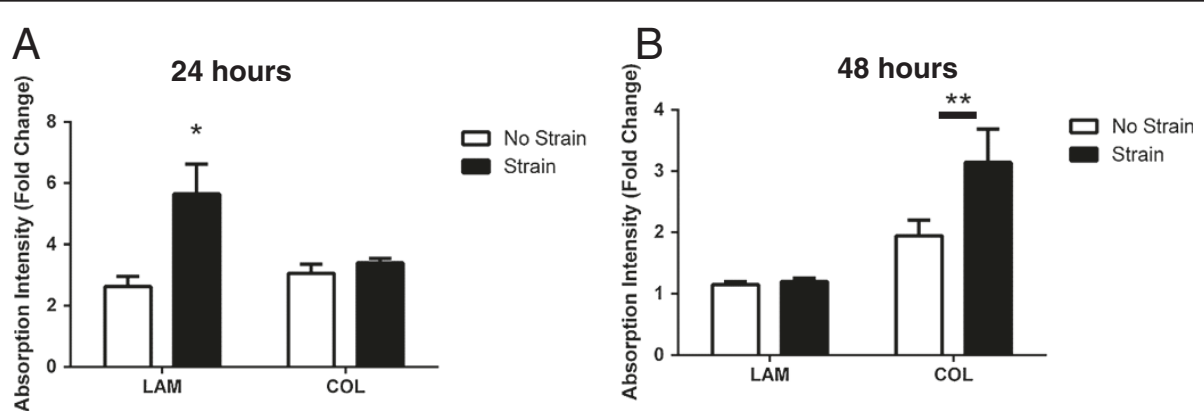

Figure 5 Myoblast proliferation after treatment with muscle-derived mesenchymal stem/stromal cell (mMSC) conditioned media. mMSCs were plated on laminin (LAM) or collagen (COL) and were either exposed to mechanical strain or remained unstrained. The quantity of primary myoblasts treated with conditioned media for 24 hours (A) or 48 hours (B) was determined by MTT assay. Data are presented as mean \pm standard error of the mean of fold change relative to myoblasts treated with serum-free media for the same amount of time. ${ }^{*} P<0.05$ versus Lam/No Strain. ${ }^{* *} P<0.01$ versus LAM with $n=6$ per group for the 24 -hour time point and $n=7$ per group for the 48-hour time point. MTT, 3-[4,5-dimethylthiazol-2-yl]-2,5-diphenyltetrazolium bromide. 

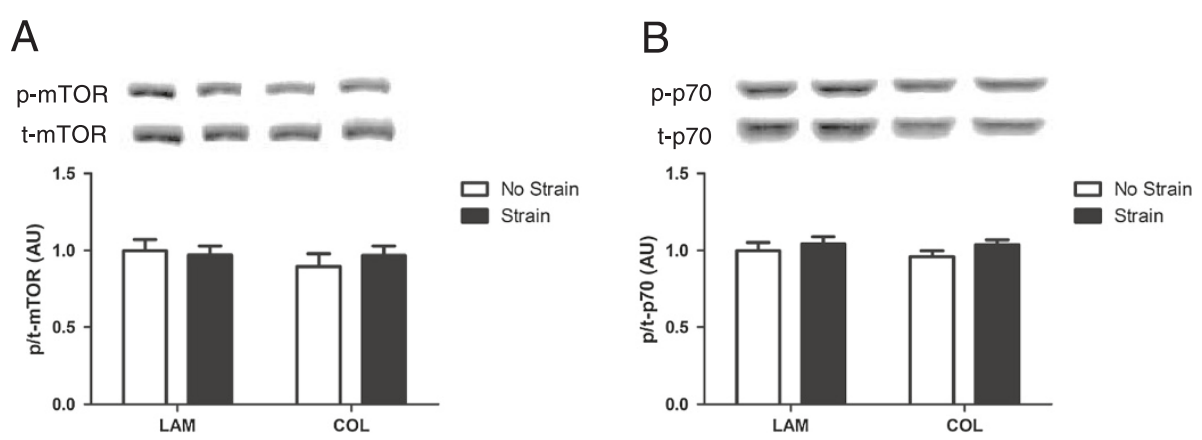

Figure 6 Hypertrophic signaling in C2C12-derived myotubes exposed to muscle-derived mesenchymal stem/stromal cell (mMSC) conditioned media. mMSCs were plated on laminin (LAM) or collagen (COL) and were either exposed to mechanical strain or remained unstrained. C2C12-derived myotubes treated with conditioned media for 3 hours were harvested and analyzed for activation of mammalian target of rapamycin (mTOR) (A) and p70 (B). Representative images are shown above each respective blot. Data are presented as mean \pm standard error of the mean. AU, arbitrary units.

to strain on LAM may indicate the early stages of ECM remodeling similar to what has been demonstrated with exercise [51]. The basally elevated levels of collagen $1 \alpha 1$ expression in mMSCs grown on COL are reminiscent of cellular dysregulation in fibrotic conditions [19,20].

A potential explanation for the decreased expression of inflammatory and ECM-related genes in mMSCs plated on collagen is that mMSCs plated on collagen recognize this collagen-rich environment as a healed wound. TGF- $\beta 1$ is well known to promote wound healing acutely, whereas excess TGF- $\beta 1$ can lead to fibrosis [52], potentially via the promotion of mMSC differentiation toward a myofibroblast phenotype [53]. Myofibroblasts are derived from a variety of cell types, including MSCs, and acute activation of myofibroblasts is important for tissue repair as they are responsible for secreting ECM proteins that form a new matrix for repaired or remodeled tissue [54]. In the present study, mMSCs plated on COL decreased their level of TGF- $\beta 1$ expression, suggesting that their differentiation toward a myofibroblast phenotype may have been impaired. These data suggest a mechanism whereby TGF- $\beta 1$ is downregulated on COL inhibiting the myofibroblast phenotype and ECM remodeling. It is important to note that chronic activation of myofibroblasts can lead to tissue fibrosis and contracture [54]. Thus, mMSCs plated on COL may recognize their environment as one that is already fully healed [52] and downregulate their expression of various repair factors, including inflammatory cytokines and ECM proteins, while plating on LAM may mimic an environment in the early stages of repair.

The alterations in mMSC gene expression in the presence of COL and LAM prompted us to investigate the capacity for the mMSC secretome to differentially impact myoblast activation and myotube growth. In vitro experiments were conducted to determine the full extent to which the mMSC secretome could impact these events in muscle. We did not observe any change in anabolic signaling (mTOR, p70) in myotubes treated with CM from mMSCs, suggesting that stimulation of protein synthesis is not the primary mechanism whereby mMSCs contribute to muscle repair or hypertrophy. On the other hand, we did observe differential regulation of myoblast proliferation in $\mathrm{CM}$ experiments. $\mathrm{CM}$ from mMSCs strained on LAM increased myoblast quantity 24 hours post-treatment relative to all other conditions. These differential effects on myoblast proliferation were seen despite no differences in HGF expression between mMSCs on LAM or COL with or without strain. HGF is the ligand for the c-met receptor and initiates satellite cell activation [55]. Since myoblasts in culture are already actively proliferating, HGF may not be the primary paracrine factor underlying this response. The impact of strain on myoblast proliferation was unexpected given the lack of change in gene expression in response to strain. However, the delayed increase in FAK phosphorylation at 24 hours post-strain in mMSCs exposed to LAM suggests that strain may have a long-term effect on intracellular actin remodeling or adhesion or both. We hypothesize that growth on LAM is a signal to increase the transcription of various growth/inflammatory factors and that mechanical strain is the stimulus to allow the release of these factors. Indeed, previous studies have shown that mechanical strain promotes release of growth factors from mMSCs [16] and MDSCs [36]. Differential production of paracrine factors in mMSCs plated on LAM versus COL may explain the upregulation of myoblast quantity at 24 hours when treated with LAM CM versus the delayed increase to 48 hours in myoblasts treated with COL CM as the concentration of paracrine factors in LAM CM may have been higher. Whether the delay in myoblast expansion observed with 
CM from COL- and strain-treated mMSCs at 48 hours is reflective of a corresponding time delay in FAK phosphorylation is not known.

In humans, NF- $\mathrm{kB}$ expression following eccentric contraction was recently linked to paracrine factor release, and NF- $\mathrm{kB}$ was localized primarily to pericytes, a population of cells similar to mMSCs [10]. Since we observed decreased inflammatory factor expression in mMSCs plated on COL in the present study, we examined the activation of NF- $\mathrm{KB}$ in mMSCs on LAM and COL with or without strain. We did not observe any alterations in levels of phosphorylated or total NF- $\mathrm{kB}$ under the conditions explored in the present investigation, suggesting that different mechanisms may be responsible for the effects observed.

A potential limitation of our study is the use of commercially available Flexcell plates pre-coated with laminin and collagen peptide. This model has been used previously in the literature to examine the effects of different substrates [31] and mechanical strain $[12,16,56]$ on primary myoblasts and mMSCs. The data presented here provide the first step in evaluating the mMSC response to strain in the context of substrate, yet further studies are necessary to confirm and expand this information. Our lab is currently developing novel methods that will allow the evaluation of the mMSC response to strain in the presence of a wide variety of full-length substrates in the context of stiffness. These studies are essential to fully understand the role for mMSCs in tissue health and assist in the development of stem cellbased therapies.

The data presented here demonstrate that MSCs are responsive to their microenvironment and this may suggest that injecting MSCs into an inflamed or fibrotic environment could alter their secretome and impair their capacity for regeneration. Previous literature would suggest that the MSC secretome is maintained for at least a short while after transplantation into an inflamed or fibrotic environment. MSC co-transplantation with hematopoietic stem cells in a hematopoietic stem cell transplant promotes hematopoietic regeneration although donor MSCs are not detected in recipient bone marrow at late time points following transplantation $[57,58]$. In cardiac infarction models, MSCs promote cardiac remodeling through secretion of paracrine factors detectable 2 weeks post-transplantation [59] although the majority of MSCs die within 4 days of transplantation [60]. Together, these studies suggest that MSCs are rapidly lost following transplantation and this may represent a protective mechanism that prevents their secretome from being altered when transplanted into an inflamed or fibrotic environment. The beneficial effects of MSC therapy in these models are realized acutely after MSC transplantation that initiates a local regenerative response through release of paracrine factors.

\section{Conclusions}

mMSCs are responsive to their environment and may participate in the detrimental changes in tissue health in fibrotic conditions. Growth on collagen 1 inhibits gene expression and alters the secretion of factors involved in myoblast proliferation or survival or both. Further studies should focus on the extent to which MSC preconditioning with laminin or mechanical strain or both can improve outcomes associated with MSC transplantation as well as determine the extent to which laminin or physical activity or both can directly recover endogenous mMSC function in diseased and aged skeletal muscle.

\section{Abbreviations}

$\mathrm{CM}$ : conditioned media; $\mathrm{CO}_{2}$ : carbon dioxide; $\mathrm{COL}$ : collagen; DMEM: Dulbecco's modified Eagle's medium; ECM: extracellular matrix; FAK: focal adhesion kinase; FBS: fetal bovine serum; GM-CSF: granulocytemacrophage colony-stimulating factor; HGF: hepatocyte growth factor; IL-6: interleukin-6; LAM: laminin; LM-111: laminin-111; MDSC: muscle-derived stem cell; mMSC: muscle-derived mesenchymal stem/stromal cell; MRF: myogenic regulatory factor; MSC: mesenchymal stem/stromal cell; mTOR: mammalian target of rapamycin; NF-KB: nuclear factor-kappa B; NSC: collagen-No Strain; NSL: laminin-No Strain; PCR: polymerase chain reaction; qPCR: quantitative polymerase chain reaction; SC: collagen 1-Strain; SFM: serum-free media; SL: laminin-Strain; TGF- $\beta 1$ : transforming growth factor-beta 1; TNF-a: tumor necrosis factor-alpha; VEGF: vascular endothelial growth factor.

\section{Competing interests}

The authors declare that they have no competing interests.

\section{Authors' contributions}

MD helped to design the study, write the manuscript, and complete experiments and analyzed the data. MB helped to design the study and write the manuscript. TJ, RAS, and HDH helped to complete experiments. All authors read and approved the final manuscript.

\section{Acknowledgments}

This work was supported by a grant from the Ellison Medical Foundation (AG-NS-0547-09 to MB). HDH was supported by a National Science Foundation Integrative Graduate Education and Research Traineeship in Cellular and Molecular Mechanics and BioNanotechnology.

\section{Author details}

'Department of Kinesiology and Community Health, University of Illinois, Urbana, IL, USA. ${ }^{2}$ Beckman Institute for Advanced Science and Technology, University of Illinois, $405 \mathrm{~N}$. Mathews Avenue, MC-251, Urbana, IL 61801, USA. ${ }^{3}$ Division of Biomedical Sciences, University of Illinois, Urbana, IL, USA.

Received: 21 February 2014 Revised: 2 June 2014

Accepted: 3 June 2014 Published: 6 June 2014

\section{References}

1. Dominici M, Le Blanc K, Mueller I, Slaper-Cortenbach I, Marini F, Krause D, Deans R, Keating A, Prockop D, Horwitz E: Minimal criteria for defining multipotent mesenchymal stromal cells. The International Society for Cellular Therapy position statement. Cytotherapy 2006, 8:315-317.

2. Keating A: Mesenchymal stromal cells. Curr Opin Hematol 2006, 13:419-425.

3. Ranganath SH, Levy O, Inamdar MS, Karp JM: Harnessing the mesenchymal stem cell secretome for the treatment of cardiovascular disease. Cell Stem Cell 2012, 10:244-258.

4. Kinnaird T, Stabile E, Burnett MS, Lee CW, Barr S, Fuchs S, Epstein SE: Marrow-derived stromal cells express genes encoding a broad spectrum of arteriogenic cytokines and promote in vitro and in vivo arteriogenesis through paracrine mechanisms. Circ Res 2004, 94:678-685.

5. Castillo-Melendez M, Yawno T, Jenkin G, Miller SL: Stem cell therapy to protect and repair the developing brain: a review of mechanisms of 
action of cord blood and amnion epithelial derived cells. Front Neurosci 2013, 7:194.

6. Valtieri $M$, Sorrentino A: The mesenchymal stromal cell contribution to homeostasis. J Cell Physio/ 2008, 217:296-300.

7. Boppart MD, De Lisio M, Zou K, Huntsman HD: Defining a role for nonsatellite stem cells in the regulation of muscle repair following exercise. Front Physiol 2013, 4:310.

8. Asakura A, Seale P, Girgis-Gabardo A, Rudnicki MA: Myogenic specification of side population cells in skeletal muscle. J Cell Biol 2002, 159:123-134.

9. Majka SM, Jackson KA, Kienstra KA, Majesky MW, Goodell MA, Hirschi KK: Distinct progenitor populations in skeletal muscle are bone marrow derived and exhibit different cell fates during vascular regeneration. J Clin Invest 2003, 111:71-79.

10. Hyldahl RD, Xin L, Hubal MJ, Moeckel-Cole S, Chipkin S, Clarkson PM: Activation of nuclear factor-KB following muscle eccentric contractions in humans is localized primarily to skeletal muscle-residing pericytes. FASEB J 2011, 25:2956-2966.

11. Lavasani M, Lu A, Thompson SD, Robbins PD, Huard J, Niedernhofer LJ: Isolation of muscle-derived stem/progenitor cells based on adhesion characteristics to collagen-coated surfaces. Methods Mol Biol Clifton NJ 2013, 976:53-65.

12. Valero MC, Huntsman HD, Liu J, Zou K, Boppart MD: Eccentric exercise facilitates mesenchymal stem cell appearance in skeletal muscle. PLoS One 2012, 7:e29760.

13. Uezumi A, Fukada S, Yamamoto N, Takeda S, Tsuchida K: Mesenchymal progenitors distinct from satellite cells contribute to ectopic fat cell formation in skeletal muscle. Nat Cell Biol 2010, 12:143-152.

14. Joe AW, Yi L, Natarajan A, Le Grand F, So L, Wang J, Rudnicki MA, Rossi FMV Muscle injury activates resident fibro/adipogenic progenitors that facilitate myogenesis. Nat Cell Biol 2010, 12:153-163.

15. Mitchell KJ, Pannerec A, Cadot B, Parlakian A, Besson V, Gomes ER, Marazzi $G$, Sassoon DA: Identification and characterization of a non-satellite cell muscle resident progenitor during postnatal development. Nat Cell Biol 2010, 12:257-266

16. Huntsman HD, Zachwieja N, Zou K, Ripchik P, Valero MC, De Lisio M, Boppart MD: Mesenchymal stem cells contribute to vascular growth in skeletal muscle in response to eccentric exercise. Am J Physiol Heart Circ Physiol 2013, 304:H72-H81.

17. Schraufstatter IU, Discipio RG, Khaldoyanidi S: Mesenchymal stem cells and their microenvironment. Front Biosci Landmark Ed 2011, 16:2271-2288.

18. Gillies AR, Lieber RL: Structure and function of the skeletal muscle extracellular matrix. Muscle Nerve 2011, 44:318-331.

19. Alexakis C, Partridge T, Bou-Gharios G: Implication of the satellite cell in dystrophic muscle fibrosis: a self-perpetuating mechanism of collagen overproduction. Am J Physiol Cell Physiol 2007, 293:C661-C669.

20. Goldspink G, Fernandes K, Williams PE, Wells DJ: Age-related changes in collagen gene expression in the muscles of $\mathrm{mdx}$ dystrophic and normal mice. Neuromuscul Disord NMD 1994, 4:183-191.

21. Brack AS, Conboy MJ, Roy S, Lee M, Kuo CJ, Keller C, Rando TA: Increased Wnt signaling during aging alters muscle stem cell fate and increases fibrosis. Science 2007, 317:807-810.

22. Castillo $A B$, Jacobs $C R$ : Mesenchymal stem cell mechanobiology. Curr Osteoporos Rep 2010, 8:98-104.

23. Case N, Xie Z, Sen B, Styner M, Zou M, O'Conor C, Horowitz M, Rubin J: Mechanical activation of $\beta$-catenin regulates phenotype in adult murine marrow-derived mesenchymal stem cells. J Orthop Res Off Publ Orthop Res Soc 2010, 28:1531-1538.

24. Sen B, Guilluy C, Xie Z, Case N, Styner M, Thomas J, Oguz I, Rubin C, Burridge K, Rubin J: Mechanically induced focal adhesion assembly amplifies anti-adipogenic pathways in mesenchymal stem cells. Stem Cells Dayt Ohio 2011, 29:1829-1836.

25. Luu YK, Pessin JE, Judex S, Rubin J, Rubin CT: Mechanical signals as a non-invasive means to influence mesenchymal stem cell fate, promoting bone and suppressing the fat phenotype. Bonekey Osteovision 2009, 6:132-149.

26. Boppart MD, Burkin DJ, Kaufman SJ: a7 $\beta 1$-Integrin regulates mechanotransduction and prevents skeletal muscle injury. Am J Physiol Cell Physiol 2006, 290:C1660-C1665.

27. Burkin DJ, Wallace GQ, Nicol KJ, Kaufman DJ, Kaufman SJ: Enhanced expression of the alpha 7 beta 1 integrin reduces muscular dystrophy and restores viability in dystrophic mice. J Cell Biol 2001, 152:1207-1218.
28. Livak KJ, Schmittgen TD: Analysis of relative gene expression data using real-time quantitative PCR and the 2(-Delta Delta C(T)) method. Methods San Diego Calif 2001, 25:402-408

29. Springer ML, Rando TA, Blau HM: Gene delivery to muscle. In Curr Protoc Hum Genet. Edited by Board Jonathan Haines Al. ; 2002. Chapter 13: Unit 13.4.

30. Huang $\mathrm{C}-\mathrm{H}$, Chen $\mathrm{M}-\mathrm{H}$, Young $\mathrm{T}-\mathrm{H}$, Jeng $\mathrm{J}-\mathrm{H}$, Chen $\mathrm{Y}-\mathrm{J}$ : Interactive effects of mechanical stretching and extracellular matrix proteins on initiating osteogenic differentiation of human mesenchymal stem cells. J Cell Biochem 2009, 108:1263-1273.

31. Wilschut KJ, Haagsman HP, Roelen BA: Extracellular matrix components direct porcine muscle stem cell behavior. Exp Cell Res 2010, 316:341-352.

32. de la Garza-Rodea AS, van der Velde-van DI, Boersma H, Gonçalves MA, van Bekkum DW, de Vries AA, Knaän-Shanzer S: Myogenic properties of human mesenchymal stem cells derived from three different sources. Cell Transplant 2012, 21:153-173.

33. Salasznyk RM, Klees RF, Hughlock MK, Plopper GE: ERK signaling pathways regulate the osteogenic differentiation of human mesenchymal stem cells on collagen I and vitronectin. Cell Commun Adhes 2004, 11:137-153.

34. Lai W-T, Krishnappa V, Phinney DG: Fibroblast growth factor 2 (Fgf2) inhibits differentiation of mesenchymal stem cells by inducing Twist2 and Spry4, blocking extracellular regulated kinase activation, and altering Fgf receptor expression levels. Stem Cells Dayt Ohio 2011, 29:1102-1111.

35. Dezawa M, Ishikawa H, Itokazu Y, Yoshihara T, Hoshino M, Takeda S, Ide C, Nabeshima $Y$ : Bone marrow stromal cells generate muscle cells and repair muscle degeneration. Science 2005, 309:314-317.

36. Beckman SA, Chen WC, Tang Y, Proto JD, Mlakar L, Wang B, Huard J: Beneficial effect of mechanical stimulation on the regenerative potential of muscle-derived stem cells is lost by inhibiting vascular endothelial growth factor. Arterioscler Thromb Vasc Biol 2013, 33:2004-2012.

37. Serrano AL, Baeza-Raja B, Perdiguero E, Jardí M, Muñoz-Cánoves P: Interleukin-6 is an essential regulator of satellite cell-mediated skeletal muscle hypertrophy. Cell Metab 2008, 7:33-44

38. Baeza-Raja B, Muñoz-Cánoves P: p38 MAPK-induced nuclear factor-kappaB activity is required for skeletal muscle differentiation: role of interleukin6. Mol Biol Cell 2004, 15:2013-2026.

39. McKay BR, De Lisio M, Johnston AP, O'Reilly CE, Phillips SM, Tarnopolsky MA, Parise G: Association of interleukin- 6 signalling with the muscle stem cell response following muscle-lengthening contractions in humans. PLOS One 2009, 4:e6027.

40. Toth KG, McKay BR, De Lisio M, Little JP, Tarnopolsky MA, Parise G: IL-6 induced STAT3 signalling is associated with the proliferation of human muscle satellite cells following acute muscle damage. PLoS One 2011, 6:e17392.

41. McKay BR, Ogborn DI, Baker JM, Toth KG, Tarnopolsky MA, Parise G: Elevated SOCS3 and altered IL-6 signaling is associated with age-related human muscle stem cell dysfunction. Am J Physiol Cell Physiol 2013, 304:C717-C728.

42. Li Y-P: TNF-alpha is a mitogen in skeletal muscle. Am J Physiol Cell Physiol 2003, 285:C370-C376.

43. Muñoz-Cánoves P, Scheele C, Pedersen BK, Serrano AL: IL-6 myokine signaling in skeletal muscle: a double-edged sword? FEBS J 2013, 280:4131-4148.

44. Carson JA, Baltgalvis KA: Interleukin 6 as a key regulator of muscle mass during cachexia. Exerc Sport Sci Rev 2010, 38:168-176.

45. Beyer I, Mets T, Bautmans I: Chronic low-grade inflammation and age-related sarcopenia. Curr Opin Clin Nutr Metab Care 2012, 15:12-22.

46. Peterson JM, Pizza FX: Cytokines derived from cultured skeletal muscle cells after mechanical strain promote neutrophil chemotaxis in vitro. J Appl Physiol Bethesda Md 1985 2009, 106:130-137.

47. Gullberg D, Tiger CF, Velling T: Laminins during muscle development and in muscular dystrophies. Cell Mol Life Sci CMLS 1999, 56:442-460.

48. Rooney JE, Gurpur PB, Burkin DJ: Laminin-111 protein therapy prevents muscle disease in the mdx mouse model for Duchenne muscular dystrophy. Proc Natl Acad Sci U S A 2009, 106:7991-7996.

49. Rooney JE, Knapp JR, Hodges BL, Wuebbles RD, Burkin DJ: Laminin-111 protein therapy reduces muscle pathology and improves viability of a mouse model of merosin-deficient congenital muscular dystrophy. Am J Pathol 2012, 180:1593-1602.

50. Goudenege S, Lamarre Y, Dumont N, Rousseau J, Frenette J, Skuk D, Tremblay JP: Laminin-111: a potential therapeutic agent for Duchenne muscular dystrophy. Mol Ther J Am Soc Gene Ther 2010, 18:2155-2163. 
51. Heinemeier KM, Olesen JL, Haddad F, Langberg H, Kjaer M, Baldwin KM, Schjerling P: Expression of collagen and related growth factors in rat tendon and skeletal muscle in response to specific contraction types. J Physiol 2007, 582:1303-1316.

52. Pakyari M, Farrokhi A, Maharlooei MK, Ghahary A: Critical role of transforming growth factor beta in different phases of wound healing. Adv Wound Care 2013, 2:215-224.

53. Hinz B: Tissue stiffness, latent TGF-beta1 activation, and mechanical signal transduction: implications for the pathogenesis and treatment of fibrosis. Curr Rheumatol Rep 2009, 11:120-126.

54. Hinz B: The myofibroblast: paradigm for a mechanically active cell. J Biomech 2010, 43:146-155.

55. Tatsumi R, Anderson JE, Nevoret CJ, Halevy O, Allen RE: HGF/SF is present in normal adult skeletal muscle and is capable of activating satellite cells. Dev Biol 1998, 194:114-128.

56. Johnston AP, Baker J, De Lisio M, Parise G: Skeletal muscle myoblasts possess a stretch-responsive local angiotensin signalling system. J Renin Angiotensin Aldosterone Syst 2011, 12:75-84.

57. Noort WA, Kruisselbrink AB, in't Anker PS, Kruger M, van Bezooijen RL, de Paus RA, Heemskerk MH, Löwik CW, Falkenburg JH, Willemze R, Fibbe WE: Mesenchymal stem cells promote engraftment of human umbilical cord blood-derived CD34(+) cells in NOD/SCID mice. Exp Hematol 2002, 30(8):870-378.

58. Bensidhoum M, Chapel A, Francois S, Demarquay C, Mazurier C, Fouillard L, Bouchet S, Bertho JM, Gourmelon P, Aigueperse J, Charbord P, Gorin NC, Thierry D, Lopez M: Homing of in vitro expanded Stro-1- or Stro-1+ human mesenchymal stem cells into the NOD/SCID mouse and their role in supporting human CD34 cell engraftment. Blood 2004, 103(9):3313-3319.

59. Tang $\sqcup$, Gao Y, Zhang Z, Li H, Shan YQ: Human bone marrow multipotent adult progenitor cells differentiate into hepatocyte-like cells with hepatocyte growth factor plus fibroblast growth factor-4 in vitro [Article in Chinese] Zhonghua Gan Zang Bing Za Zhi 2005, 13(9):652-655.

60. Pons J, Huang Y, Takagawa J, Arakawa-Hoyt J, Ye J, Grossman W, Kan YW, Su H: Combining angiogenic gene and stem cell therapies for myocardial infarction. J Gene Med 2009, 11(9):743-753.

\section{doi:10.1186/scrt463}

Cite this article as: De Lisio et al.: Substrate and strain alter the muscle-derived mesenchymal stem cell secretome to promote myogenesis. Stem Cell Research \& Therapy 2014 5:74.

\section{Submit your next manuscript to BioMed Central and take full advantage of:}

- Convenient online submission

- Thorough peer review

- No space constraints or color figure charges

- Immediate publication on acceptance

- Inclusion in PubMed, CAS, Scopus and Google Scholar

- Research which is freely available for redistribution 